\title{
Impact Des Facteurs De Pollution Sur La Qualite Des Eaux De La Zone Aval De La Vallee De L'oued Nekor (Al-Hoceima, Maroc)
}

\author{
Fouad Dimane, Prof. \\ Khadija Haboubi, Prof. \\ Issam Hanafi, Prof. \\ Abdelouahad El Himri, Prof. \\ Laboratory of Engineering Sciences and Applications, \\ National Schoolof Applied Sciences Al Hoceima (ENSAH) \\ Mohammed Premier University, Morocco \\ Kaouthar Andaloussi, Dr. \\ Laboratoire de l'Eau, d'Etudes et des Analyses Environnementales \\ Département de chimie, Faculté des sciences \\ Université Abdelmalek Essaâdi-Tétouan - Maroc
}

doi: 10.19044/esj.2016.v13n3p43 URL:http://dx.doi.org/10.19044/esj.2016.v13n3p43

\begin{abstract}
Water is a precious and essential natural resource for multiple uses. Its use for food or hygiene requires excellent physicochemical and microbiological quality. To assess the quality of well water for human consumption in the Al Hoceima region which is located in North of Morocco, a physico-chemical and bacteriological control was carried out on the basis of 10 water samples from wells in this area. The analyzes were performed on these samples by measuring the following physic-chemical parameters: temperature, $\mathrm{pH}$, electric conductivity (EC), chloride $\left(\mathrm{Cl}^{-}\right)$, dissolved oxygen, nitrate $\left(\mathrm{NO}_{3}{ }^{-}\right)$, and possibly seeking unwanted bacteria (total bacteria, total coliforms, fecal coliforms, fecal streptococci). The samples were analyzed systematically to measure all the physicochemical indicators of pollution. Correlation between pollution parameters were conducted to give a reponse element on the main causes pollution. The results performed analysis, revealed a pollution of an agricultural origin. This is confirmed by the maximum levels of chlorides, which exceed $2640 \mathrm{mg} / \mathrm{l}$, thereby explaining the high conductivity levels $(4800 \mu \mathrm{s} / \mathrm{cm})$. In addition $14 \%$ of analyzed wells have nitre levels $50 \mathrm{mg} / \mathrm{l}$ nitrate pollution a not alarming. However, if a pollution of agricultural origin is mentioned, the lithological contribution can not be neglected on the results obtained.
\end{abstract}


Keywords: Groundwater, Physicochemical Parameters, Pollution, Quality, Al Hoceima

\section{Résumé}

L'eau est une ressource naturelle précieuse et essentielle pour de multiples usages. Son utilisation à des fins alimentaires ou d'hygiène nécessite une excellente qualité physico-chimique et microbiologique. Pour apprécier la qualité des eaux de puits destinées à la consommation humaine dans la plaine de Rhiss-Nekor qui est située au Nord du Maroc, un contrôle a été réalisé et a porté sur plusieurs échantillons d'eau prélevés au niveau de 10 puits appartenant à cette localité. Les échantillons prélevés ont été systématiquement analysés en vue de mesurer tous les paramètres indicateurs de pollution : la conductivité électrique (CE), les chlorures $\left(\mathrm{Cl}^{-}\right)$, l'oxygène dissous, les nitrates $\left(\mathrm{NO}_{3}{ }^{-}\right)$, et en recherchant éventuellement les germes indésirables (coliformes totaux, coliformes fécaux, streptocoques fécaux). Des corrélations entre ces paramètres ont été effectuées afin de donner des éléments de réponse aux causes principales de pollution. Les résultats des analyses effectuées, ont révélé une pollution chlorée des eaux de la nappe. Ceci est confirmé par des teneurs élevées en chlorures qui dépassent les 2840mg/l, ce qui explique les valeurs élevées de la conductivité $(4000 \mu \mathrm{s} / \mathrm{cm})$. En outre, $14 \%$ des puits analysés ont des teneurs en nitrates supérieures à 50mg/l enregistrant une pollution nitrique qui n'est pas alarmante. Cependant, si une pollution d'origine agricole est évoquée, on ne peut négliger la contribution lithologique sur les résultats obtenus.

Mots-Clés: Eaux souterraines, Paramètres Physico-chimie, Bacteriologie, Pollution, Rhiss-Nekor

\section{Introduction}

Partout dans le monde, la pression sur les ressources en eau et en particulier sur les ressources en eau souterraines est à la hausse, principalement en raison de la demande croissante (Nouayti, 2015). Dans le contexte actuel de la politique de gestion durable des ressources en eau, la prédiction du risque de pollution et la protection de ces ressources sont d'une importance capitale. L'eau est une composante de l'environnement la plus vulnérable aux différentes contaminations, en particulier les eaux souterraines. L'eau est devenue aujourd'hui un enjeu stratégique mondial dont la gestion doit impérativement s'intégrer dans une perspective politique de développement durable (Servais, 2009). Pour cela, la sauvegarde, à moyen et à long terme, de la qualité de ces ressources s'impose (Amharref et al., 2007). 
L'impact de cette pollution sur les ressources en eau devient de plus en plus majeur. La gestion de l'eau en tant que ressource naturelle est une question préoccupante pour de nombreux états. Le rapport de l'OCDE qualifie ce problème comme nécessitant une attention urgente (OCDE, 2001).

$\mathrm{Au}$ Maroc les eaux souterraines constituent une part importante du patrimoine hydraulique du pays. La qualité des eaux de surface et souterraine a connu ces dernières années au Maroc, une grande détérioration, en raison du développement urbain et industriel (Matee, 2001). Ces eaux souterraines sont traditionnellement les ressources en eau privilégiées pour l'eau potable, car plus à l'abri des polluants que les eaux de surface (Guergazi et al., 2005). La disponibilité d'une eau de bonne qualité est un élément indispensable pour prévenir les maladies et améliorer la qualité de vie (Olduro, A.O. et Aderiye, 2007). Le défi auquel font face toutes les régions du Maroc et particulièrement les zones rurales est la protection de la qualité des ressources en eau souterraine. En effet, la pollution de ces eaux souterraines représente l'un des aspects les plus inquiétants et l'utilisation de ces eaux à des fins alimentaires représente un danger pour la santé (Laferriere et al., 1996). Au Maroc, les eaux souterraines sont polluées à partir de la surface et sont irréversiblement endommagés par l'intrusion d'eau saline. Certains traveaux de recherche ont été réalisés sur la qualité des eaux souterraines concluant que les pollutions des ces eaux proviendraient d'une origine géologique et anthropique, notamment d'infiltration des eaux usées et l'utilisation des engrais chimiques en agriculture (Boualla et al., 2011; Aka et al., 2013).

Ce travail a pour objectif d'effectuer un diagnostic de l'état de la province d'Al Hoceima (plaine de Rhiss-Nekor) et se propose de dresser un état préliminaire de la qualité des eaux après le démarrage de la station d'épuration d'Imzouren.

\section{Matériels et méthodes}

\section{Présentation de la zone d'étude}

La zone d'étude fait partie de la province d'Al-Hoceima au Nord du Maroc (figure 1) sur le littoral méditerranéen. La plaine du Rhiss-Nekor comprise entre $3^{\circ} 46^{\prime}, 3^{\circ} 49^{\prime}$ et $3^{\circ} 54^{\prime}$ de longitude Ouest et $35^{\circ} 13^{\prime}, 35^{\circ} 05^{\prime}$ et $35^{\circ} 12^{\prime}$ de latitude Nord. Cette plaine est une vallée intra-montagneuse occupée par un remplissage alluviale hétérogène constitué de sables, graviers et conglomérats du Plio-quaternaire et de l'Actuel. La plaine appartient pour $90 \%$ au Nekor, le Rhiss n'intervient que dans l'extrémité nord-ouest. De forme triangulaire, elle s'étend sur une superficie d'environ $100 \mathrm{~km}^{2}$ entre la mer Méditerranée au nord, le massif calcaire des Bokoya au nord-ouest, les vulcanites au nord-est, les cônes de déjection à l'est et par les flyschs schisto- 
gréseux imperméables au sud-est. La zone d'étude recèle deux nappes d'eau, la première dite nappe superficielle dans laquelle sont implantés tous les puits domestiques. Les eaux de ces puits sont surtout destinées à l'irrigation. La seconde nappe profonde dénommée nappe des graviers, cette nappe est captée par des forages. Les eaux sont utilisées pour l'alimentation en eau potable (AEP) et l'irrigation. La plaine Rhiss-Nekor comprend les meilleures terres cultivables et elle abrite les plus importantes concentrations de populations et les principales agglomérations dans la région d'Al-Hoceima. Elle est soumise à un climat de type méditerranéen caractérisé par un été chaud et sec et auquel succède une saison pluvieuse fraiche, et conditionné par deux facteurs: l'influence maritime et l'existence de la chaîne de montagnes du Rif.

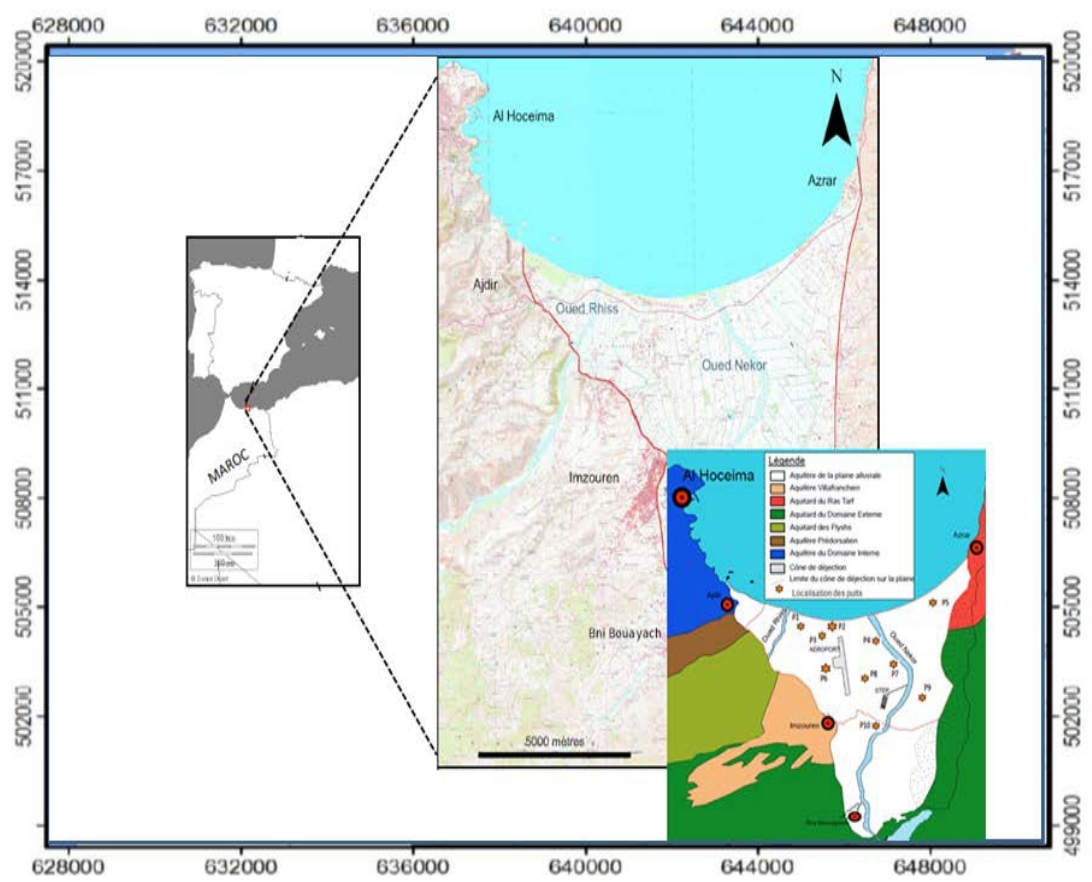

Figure 1 : localisation du site d'étude et des stations de prélèvement

\section{Echantillonnage et analyses}

\section{Prélèvement des eaux et mesures des paramètres physico-chimiques}

Pour mieux caractériser la qualité des eaux souterraines, un réseau d'échantillonnage a été choisi pour permettre d'acquérir des données représentatives sur la variabilité spatiale des éléments étudiés. Ce réseau couvre la totalité de la plaine de l'amont vers l'aval. Il se compose d'un ensemble de puits. Les prélèvements d'eau ont été effectués en période de basses eaux, effectués pendant deux campagnes du 04 juin 2015 au 20 juillet 2015. Les points d'eau ont été choisis de manière à avoir une image 
d'ensemble de la nappe phréatique du plio-quaternaire. Nous avons effectué au total dix prélèvements pour l'analyse physico-chimique et bactériologique des eaux répartis comme suit : cinq prélèvements durant la période de juin 2015 et cinq prélèvements durant la période de juillet 2015.

Les prélèvements des échantillons pour l'analyse physico-chimique ont été mis dans des flacons en plastique puis acheminés au laboratoire pour l'analyse. Les analyses physico-chimiques ont concerné les paramètres suivant: $\mathrm{T}^{\circ} \mathrm{C}, \mathrm{pH}$, Conductivité électrique, Chlorures, Sodium, l’Oxygène dissous, Sulfates et Nitrates. Les mesures des paramètres physiques (température, $\mathrm{pH}$, conductivité et l'oxygène dissous) ont été réalisées in situ par des appareils portatifs (Tableau 1), alors que les analyses chimiques des échantillons ont été effectuées au laboratoire en se référant au Manuel de (Rodier et al., 2009), et selon les méthodes mentionnées dans le Tableau 2.

Tableau 1: Méthodes et techniques utilisées pour les analyses des paramètres physiques

\begin{tabular}{|c|c|c|c|}
\hline \multirow[t]{2}{*}{ Paramètres } & \multirow[t]{2}{*}{ Unités } & \multicolumn{2}{|c|}{ Matériels et Méthodes } \\
\hline & & Matériels & $\begin{array}{l}\text { Méthodes } \\
\text { d'analyse }\end{array}$ \\
\hline $\begin{array}{l}\text { Température } \\
\text { Conductivité }\end{array}$ & $\begin{array}{c}{ }^{\circ} \mathrm{C} \\
\mu \mathrm{s} / \mathrm{cm}\end{array}$ & Conductivimètre & \multirow{3}{*}{$\begin{array}{l}\text { Réalisées in- } \\
\text { situ }\end{array}$} \\
\hline $\mathrm{pH}$ & & $\begin{array}{c}\text { pH mètre de marque } \\
\text { photometer Clph, lovibond « and } \\
\text { tintometer GmbH, 44287 Dortmund }\end{array}$ & \\
\hline Oxygène dissous & $\mathrm{mg} / \mathrm{l} \mathrm{O}$ & Oxymètre model HQ 40d & \\
\hline
\end{tabular}

Tableau 2: Méthodes et techniques utilisées pour les analyses des paramètres chimiques

\begin{tabular}{|c|c|c|c|c|}
\hline \multirow[t]{2}{*}{ Paramètres } & \multicolumn{2}{|c|}{ Matériels et Méthodes } & \multirow[t]{2}{*}{ Unités } & \multirow[t]{2}{*}{ Référence } \\
\hline & Matériels & Méthodes & & \\
\hline $\begin{array}{c}\text { Nitrates } \\
\left(\mathrm{NO}_{3}^{-}\right)\end{array}$ & \multirow{3}{*}{$\begin{array}{c}\text { Lecture } \\
\text { effectuée par } \\
\text { le } \\
\text { spectrophoto } \\
\text { mètre du } \\
\text { type } \\
\text { ANACHEH } \\
220 .\end{array}$} & \multirow{2}{*}{$\begin{array}{c}\text { méthode Salicylate } \\
\text { de sodium } \\
\text { méthode au bleu } \\
\text { d’indophénol }\end{array}$} & \multirow{3}{*}{$\mathrm{mg} / \mathrm{l}$} & \multirow{3}{*}{$\begin{array}{l}\text { (Rodier et } \\
\text { al., 2009) }\end{array}$} \\
\hline $\begin{array}{c}\text { Ammonium } \\
\left(\mathrm{NH}_{4}^{+}\right)\end{array}$ & & & & \\
\hline $\begin{array}{c}\text { Chlorures } \\
\left(\mathrm{Cl}^{-}\right)\end{array}$ & & $\begin{array}{l}\text { méthode } \\
\text { Néphélométrique } \\
\text { méthode de Mohr }\end{array}$ & & \\
\hline
\end{tabular}

\section{Prélèvements et analyses microbiologiques}

L’étude des paramètres bactériologiques a porté sur la quantification des paramètres d'origine fécale : coliformes fécaux (CF), coliformes totaux (CT) et streptocoques fécaux (SF). Les prélèvements ont été effectués d'après la procédure de prélèvement et d'analyse (ONEP, 2007). Le dénombrement des CF, CT et SF a été effectué selon la méthode indirecte de fermentation en tube multiple dans un bouillon lactosé; le nombre a été ensuite déduit statistiquement suivant la méthode du nombre le plus probable (Rodier et al., 2009). 


\section{Résultats et discussion Température $\left({ }^{\circ} \mathrm{C}\right)$}

La température de l'eau est un facteur écologique qui entraîne d'importantes répercutions écologiques (Leynaud, 1968). Les mesures insitu de la température au niveau des eaux de puits contrôlés ont donné des valeurs ne dépassant pas $25^{\circ} \mathrm{C}$ (figure 2), d'où elles sont en conformités avec les normes marocaines relatives aux eaux souterraines (NM 03.07.001, 2006). Dans la région d'étude, les résultats obtenus montrent que le degré de température ne présente pas de grandes variations d'un puits à l'autre, avec un minimum de $19,2^{\circ} \mathrm{C}$ (puits $\mathrm{P} 1, \mathrm{P} 3$ et P4) et un maximum de $24,3^{\circ} \mathrm{C}$ (puits P6). Cependant, la température des eaux de puits étudiés montre une stabilité spatiale.

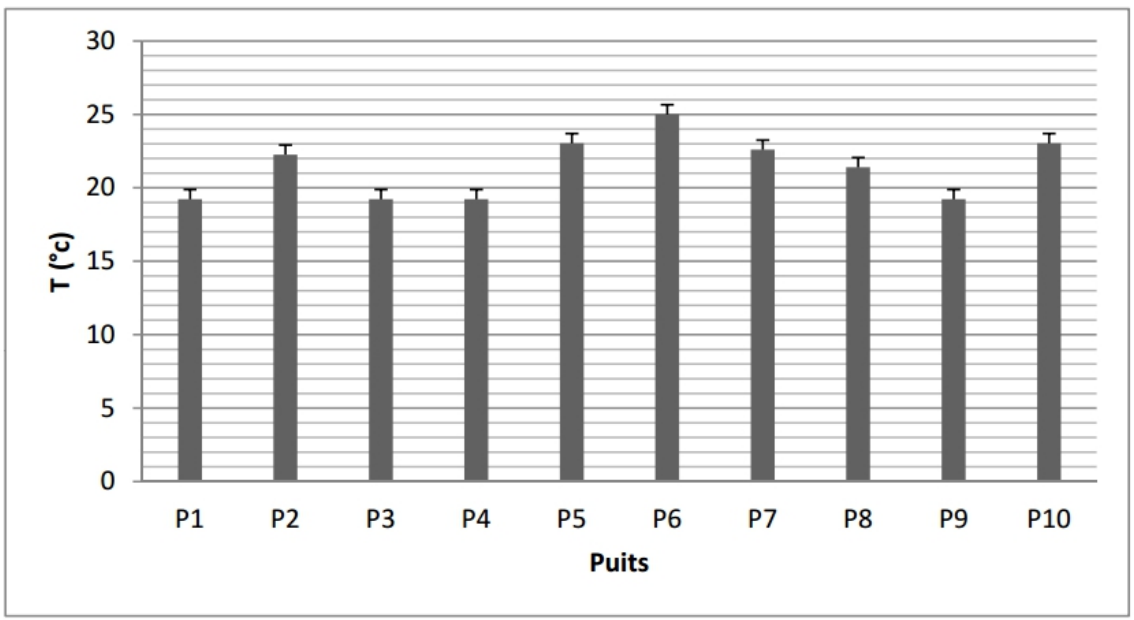

Figure 2: Variation spatiale des valeurs de la température des eaux de puits

\section{Potentiel Hydrogène (pH)}

Le $\mathrm{pH}$ de l'eau renseigne sur son acidité et son alcalinité. Selon (Nisbet et al., 1970), le $\mathrm{pH}$ des eaux naturelles est généralement compris entre 6,6 à 7,8 et selon (Rodier, 1984), il varie de 7,2 à 7,6. Habituellement, les valeurs du pH se situent entre 6 et 8,5 dans les eaux naturelles (Chapman et al.,1996). Il s'agit évidemment d'un paramètre important de caractérisation des eaux. Le pH dépend de l'origine des eaux, de la nature géologique du substrat et du bassin versant traversé (Dussart, 1966 ; Bermond et Vuichard, 1973). L'analyse des valeurs enregistrées du $\mathrm{pH}$ ne montrent pas de variations notables, avec un minimum de 6,85 (P5), un maximum de 7,85 (P8) et une moyenne de 7,12; ce qui témoigne d'une légére alcalinité (figure. 3). Au niveau de l'ensemble des points d'eau, les valeurs moyennes du $\mathrm{pH}$ au niveau de la zone d'étude ont été dans les normes de potabilité de l'eau souterraine, toutes les valeurs ont correspondu 
au pH des eaux naturelles et semble être provenant des aquifères carbonatés. Ces valeurs restent acceptables pour les normes marocaines de potabilité. (NM 03.07.001, 2006).

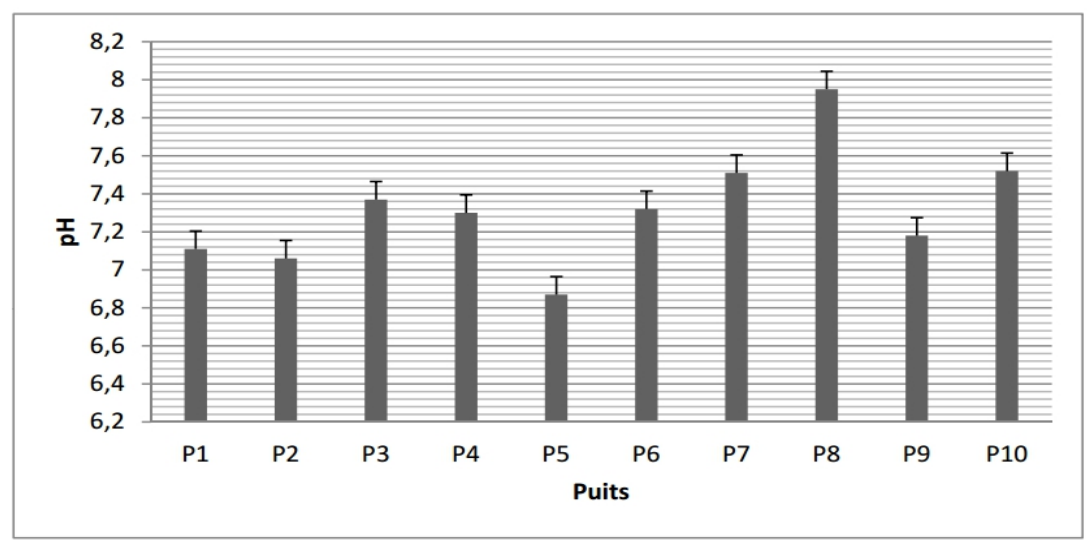

Figure. 3. Variation spatiale des valeurs moyennes de $\mathrm{pH}$

\section{La conductivité électrique des eaux}

La conductivité électrique des eaux désigne la capacité de l'eau à conduire un courant électrique. Elle est déterminée par la teneur en substances dissoutes, la charge ionique, la capacité d'ionisation, la mobilité et la température de l'eau (Derwich et al., 2010). Par conséquent, la conductivité électrique renseigne sur le degré de minéralisation d'une eau. Les eaux des points contrôlés sont minéralisées (figure. 4), avec des valeurs qui oscillaient entre minimum de $358,88 \mu \mathrm{S} / \mathrm{cm}$ (P6) et un maximum de $4897,79 \mu \mathrm{S} / \mathrm{cm}$ (P3). Toutes ces valeurs ne dépassent pas la norme marocaine de potabilité fixée à $2700 \mu \mathrm{s} / \mathrm{cm}$ (NM 03.07.001, 2006), excepté le puits (P1, P3, P4 et P10) qui affichent des valeurs trop élevées. Ces valeurs importantes semblent résulter du lessivage de la roche réservoir au sein de laquelle les eaux séjournaient.

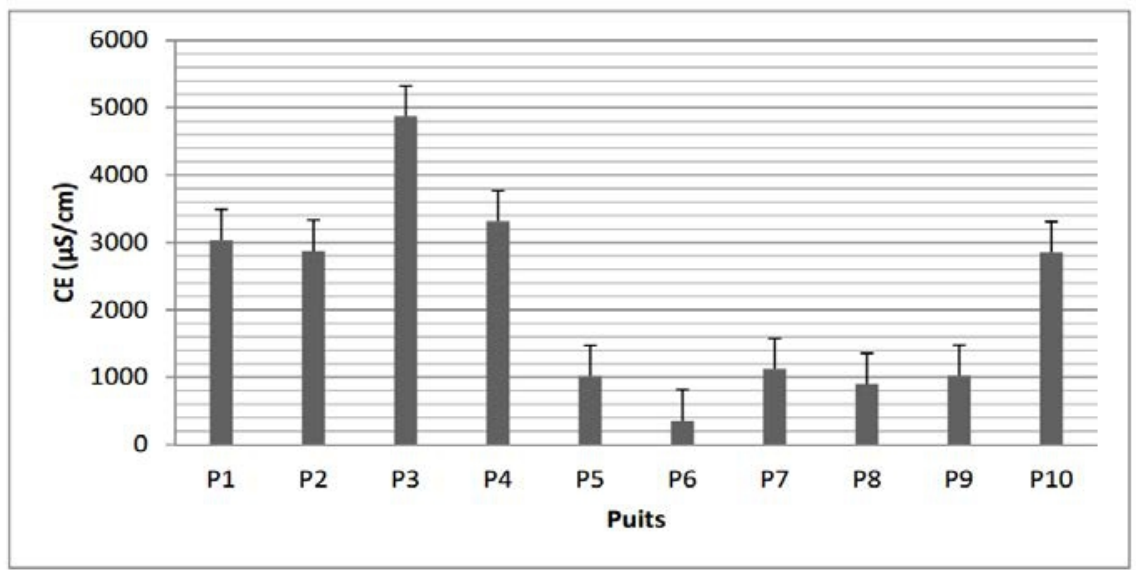

Figure 4 : Variation de la conductivité électrique des eaux de puits 


\section{L’oxygène dissous}

L’oxygène dissous (O2) est très important par le fait qu'il conditionne l'état de plusieurs sels minéraux, la dégradation de la matière organique et la vie des animaux aquatiques (HCEFLCD, 2007). Etant l'un des plus importants indicateurs du degré de pollution des eaux, l’oxygène dissous mesure la concentration du dioxygène dissous dans l'eau exprimée en mg/l ou en pourcentage de saturation. Les concentrations obtenues au niveau des eaux des différents puits étudiés ne présentent pas de variation notable. CLes eaux étudiées contienent de faibles taux d'oxygène dissous avec une valeur minimale qui atteigne $3,37 \mathrm{mg} / \mathrm{l}$ (P8) et une maximale 6,27mg/l (P1) (Figure. 5). La faible concentration des puits P7, P8 et P10 serait en relation avec les eaux polluées qui s'infiltres vers la nappe phréatique (Belghiti et al., 2013). Les résultats obtenus montrent que les puits sont légérement sous-oxygénés. Ces valeurs enregistrées de ce paramètre au niveau des puits étudiés répondent à la norme marocaine (NM 03.7.001, 2006).

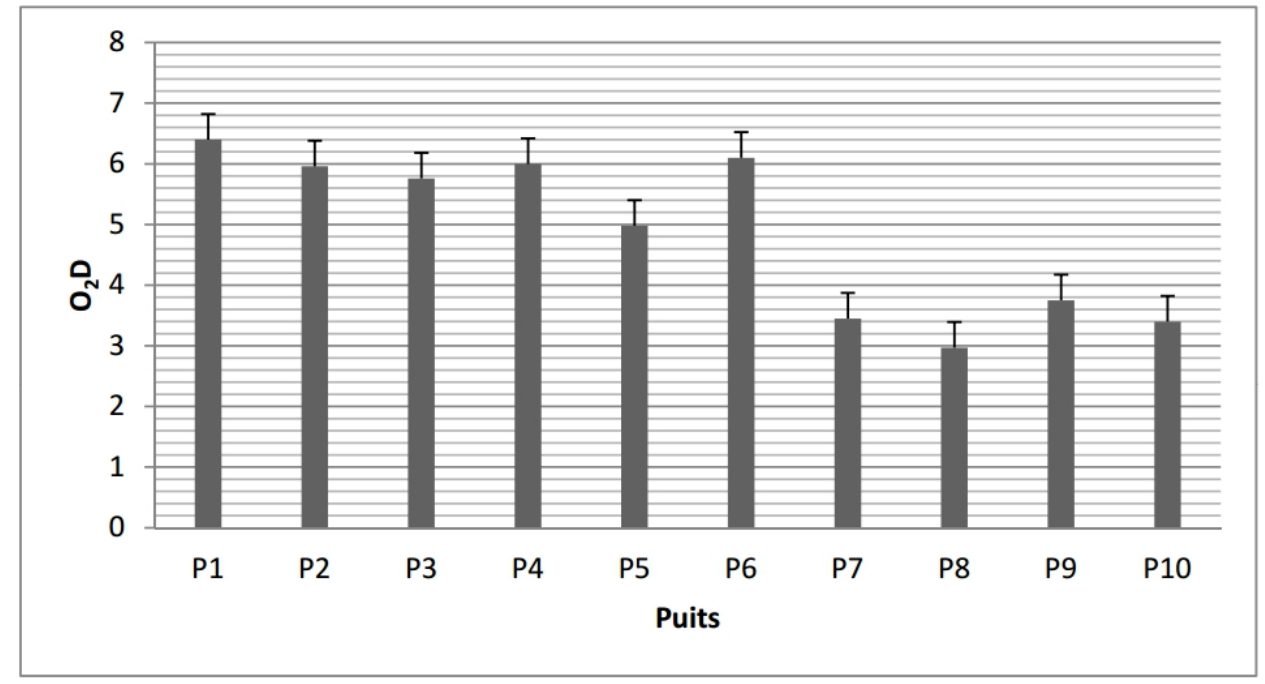

Figure 5 : Variation de l'oxygène dissout dans les eaux de puits

\section{Sulfates $\left(\mathrm{SO}_{4}{ }^{2-}\right)$}

Le sulfate naturellement présent dans l'eau à des concentrations très variables (majeure au contact du gypse ou d'autres minéraux communs) (Queneau et al., 2009). Les sulfates dans les eaux naturelles, ont essentiellement deux origines : géochimique et atmosphérique (Deliste et al., 1977). Ils résultent également de l'activité de certaines bactéries (chlorothiobactéries, rhodothiobactéries, etc.). Cette activité peut oxyder l’hydrogène sulfuré $\left(\mathrm{H}_{2} \mathrm{~S}\right)$ toxique en sulfate (HCEFLCD, 2006). 
Les valeurs des sulfates dans les eaux étudiées sont très variables, elles oscillaient entre 120,9 mg/l à 673,56 mg/l (figure.6). P5 avait le niveau de sulfate le moins élevé alors que P8 avait le niveau le plus élevé qui dépasse la valeur de la norme. La valeur moyenne du niveau de sulfate pour les échantillons analysés était de 108,26mg/l. Presque tous les résultats confirment l'acceptabilité de ces eaux souterraines en termes de leur teneur en sulfates. Selon cette étude, $75,5 \%$ des puits de la région d'étude sont conformes aux normes OMS.

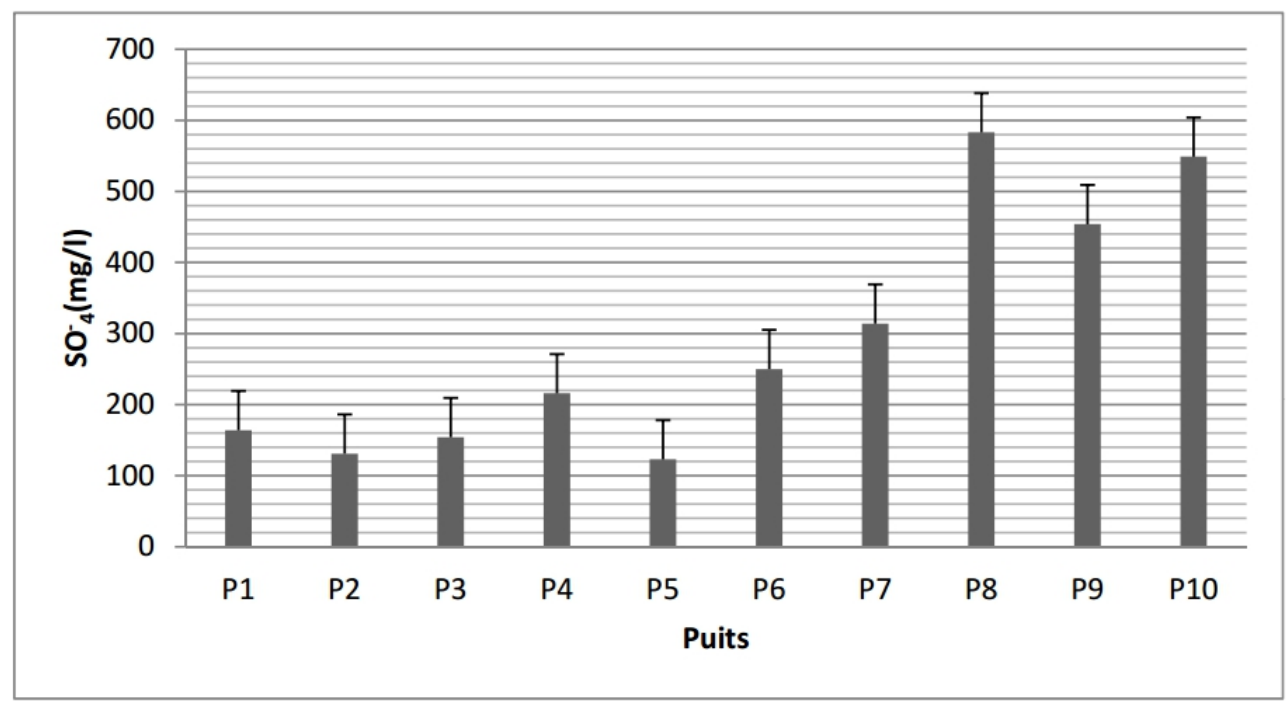

Figure 6 : Variation spatiale des teneurs en sulfates dans les eaux de puits

\section{Chlorures (CL)}

La présence de chlorures dans les eaux est due le plus souvent à la nature des terrains traversés. On les trouve dans presque toutes les eaux naturelles (Deghbey, 2011). Ces ions chlorures proviennent de lentilles argileuses présentes dans le bassin, elles peuvent également être en partie d'origine humaine (Gaujous, 1995). Les teneurs en chlorures des échantillons d'eau analysés (figure.7) affichaient des valeurs oscillant entre $150 \mathrm{mg} / \mathrm{L}$ et $2565,7 \mathrm{mg} / \mathrm{l}$. Les points d'eau situés en amont de la plaine, sont conformes aux normes, étant donné que la concentration en chlorures est inférieure à celle recommandée par les normes marocaines dans le cas des eaux souterraines et qui est de l'ordre de $750 \mathrm{mg} / \mathrm{l}$. En aval de la plaine, les points P1 et P3 renfermaient des teneurs en chlorures qui dépassaient les normes standards. 


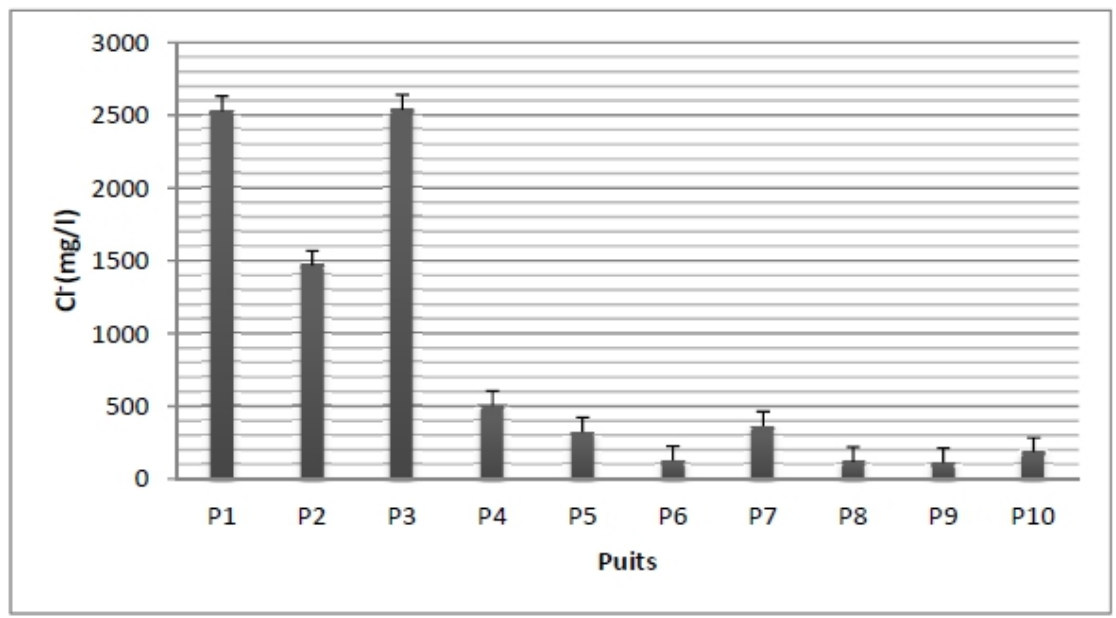

Figure 7: Variation spatiale des concentrations moyennes en chlorures

\section{Sodium $\left(\mathrm{Na}^{+}\right)$}

L'analyse des données a montré que les teneurs moyennes en sodium dans les eaux des points étudiés variaient de $26 \mathrm{mg} / \mathrm{l}$ à 350,2 mg/l (figure.8). Nous avons remarqué que les points situés en aval dépassaient les normes de potabilités. L'augmentation du sodium dans les eaux souterraines à des concentrations supérieures à la norme peut indiquer la présence d'agents polluants ou une invasion d'eau salée. Les teneurs élevées en sodium des eaux de la nappe de Rhiss-Nekor ont la même origine que celle des chlorures. En effet, le sodium accompagne généralement la pollution due aux chlores. La figure 9, montre une bonne corrélation linéaire positive entre les deux éléments en question. Il y a toujours corrélation linéaire entre ces deux éléments (Pesson, 1976).

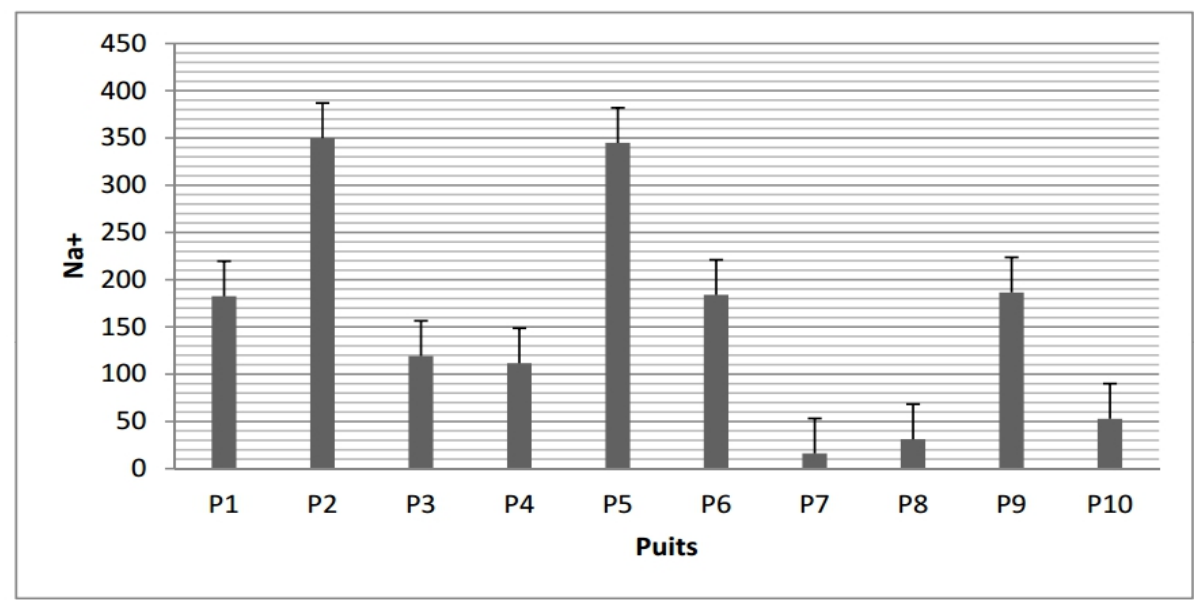

Figure 8 : Variation spatiale des concentrations moyennes en sodium 


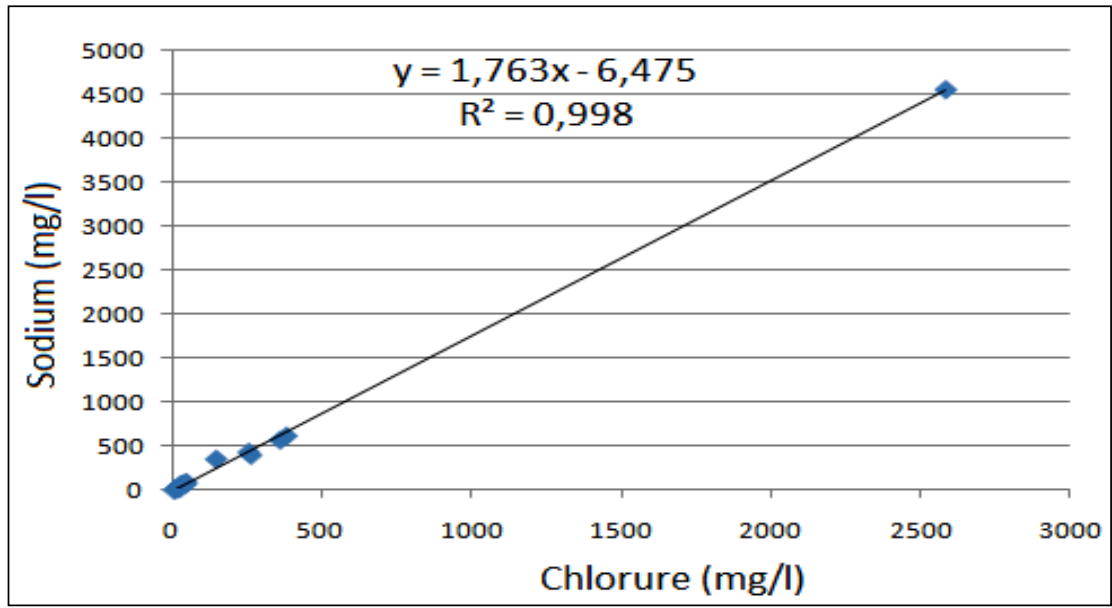

Figure 9 : Courbe de corrélation entre les concentrations des chlorures et du sodium des eaux souterraines.

\section{Répartition des Nitrates $\left(\mathrm{NO}_{3}{ }^{-}\right)$}

Les nitrates constituent le stade final de l'oxydation de l'azote organique, leur présence dans une eau polluée atteste que le processus d'autoépuration est déjà entamé. L’activité humaine accélère le processus d'enrichissement en cet élément sur les sols subissant l'érosion, ce qui provoque l'infiltration des eaux usées, par les rejets des industries minérales et d'engrais azoté (Bermond et al., 1979). La répartition des teneurs en nitrates en amont de la plaine est liée aux facteurs polluants, et en particulier à l'activité agricole et à la nature lithologique de la couche superficielle. (Boualla et al., 2011).

Dans les stations étudiées et comme il est indiqué sur la figure 10 les teneurs en nitrates variaient entre $12,89 \mathrm{mg} / \mathrm{l}$ et $102 \mathrm{mg} / \mathrm{l}$. Elles n'ont pas dépassé les normes marocaines, sauf pour les puits P8, P9 et P10. Les valeurs les plus élevées sont enregistrées en amont de la plaine au voisinage de la staion d'épuration des eaux usées. Cette dégradation semble être due essentiellement à l'activité humaine, la fertilisation azotée des zones agricoles avoisinantes aux points étudiés, les eaux usées, les puits perdus et les déchets des élevages. Les résultats obtenus sont similaires aux conclusions dégagées dans le cas de basse Moulouya confirmant l'origine anthropique des nitrates (Brahimi et al., 2014). 


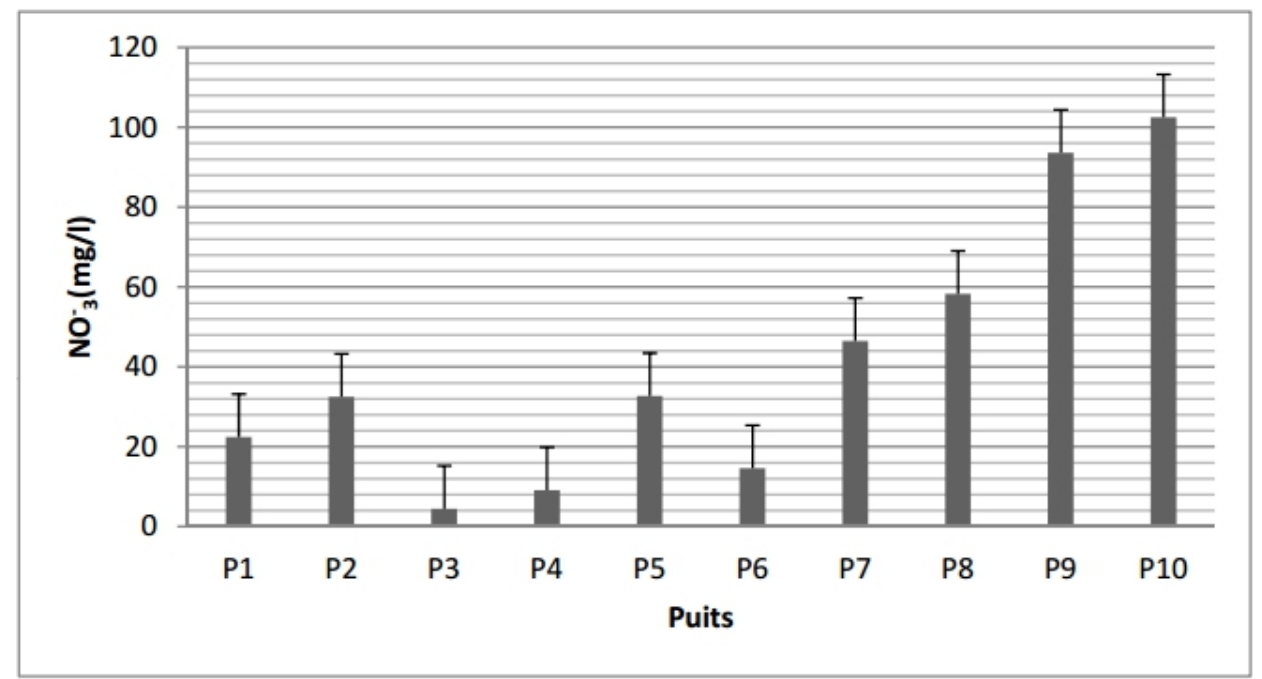

Figure 10: Variation des moyennes en Nitrate des eaux de puits

\section{Répartition de l'azote ammoniacal $\left(\mathrm{NH}_{4}{ }^{+}\right)$}

L'ammonium constitue le produit de la réduction finale des substances organiques azotées et de la matière inorganique dans les eaux et les sols. Il provient également de l'excrétion des organismes vivants et de la réduction et la biodégradation des déchets, sans négliger les apports d'origine domestique, industrielle et agricole. Cet élément existe en faible proportion inférieure à $0,1 \mathrm{mg} / \mathrm{l}$ d'azote ammoniacal dans les eaux naturelles. Dans les eaux superficielles, il provient de la matière organique azotée, et des échanges gazeux entre l'eau et l'atmosphère (Chapman et al., 1996). Il constitue ainsi un bon indicateur de la pollution des cours d'eau par les effluents. Les concentrations en ammonium sont en dessous de la norme marocaine $(0.5 \mathrm{mg} / \mathrm{l})$ dans les puits situés dans la zone d'étude. Un seul puits (P4) présente une valeur $(0,84 \mathrm{mg} / \mathrm{l})$ (figure.11) dépassant la norme marocaine et celle de l'OMS, et qui normalement doit être limitée à une valeur inférieure à $0,5 \mathrm{mg} / \mathrm{l}$. Cette pollution peut être due à la décomposition des déchets azotés (urées, azote organique) et/ou le non respect des périmètres de protection puisque en amont des puits (P3 et $\mathrm{P} 4$ ), il y a des dépôts de fumier. 


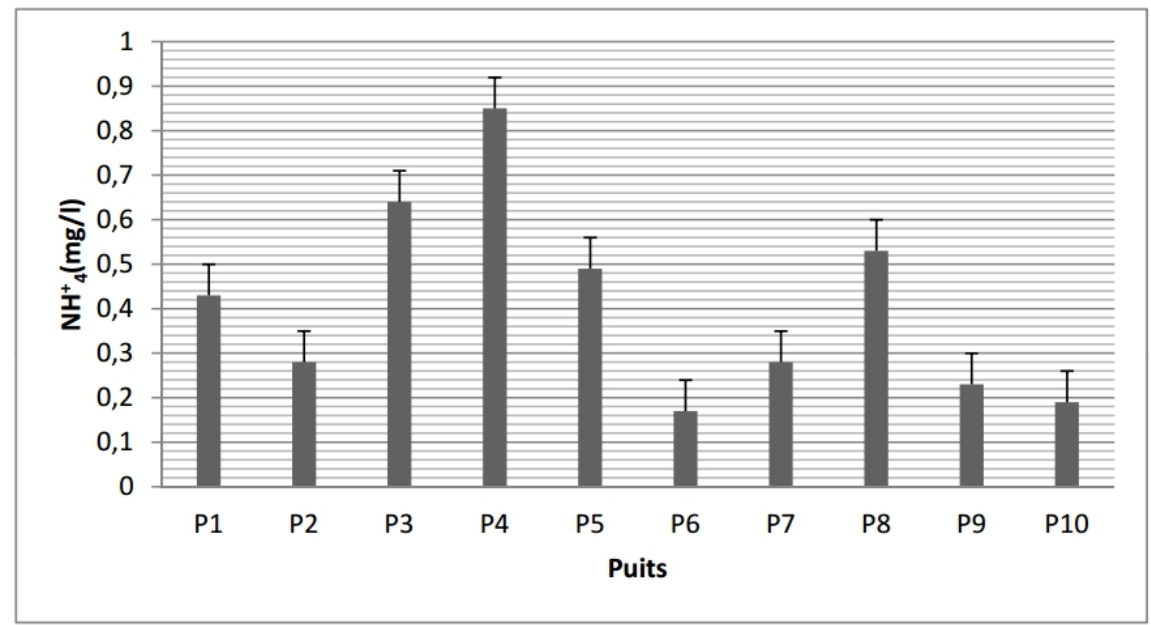

Figure 11: Variation des moyennes Ammonium des eaux de puits

\section{Teneurs en phosphates}

Les orthophosphates constituent la forme prédominante du phosphore disponible dans l'eau. En combinaison avec l'azote, le phosphore est utilisé dans les engrais chimiques et naturels pour enrichir les sols agricoles. Au cours des dernières années, une attention particulière a été apportée à cet élément afin de mieux contrôler la fertilisation agricole (Polan et al., 1990). Les teneurs des orthophosphates dans les puits étudiés varient entre une valeur minimale de $0.002 \mathrm{mg} / \mathrm{l}$ et une maximale de 0,235 mg/l (P9) (Figure. 12). Ces valeurs restent très faibles et inférieures à la valeur maximale admissible fixée à $0,5 \mathrm{mg} / \mathrm{l}$. Ces teneurs, très faibles à nulles, laissent prédire que cet élément ne constitue pas un risque de pollution pour les eaux de la nappe alluviale étudiée. Ceci pourrait s'expliquer par le fait que les orthophosphates sont facilement fixés par le sol (Rodier et al., 1984).

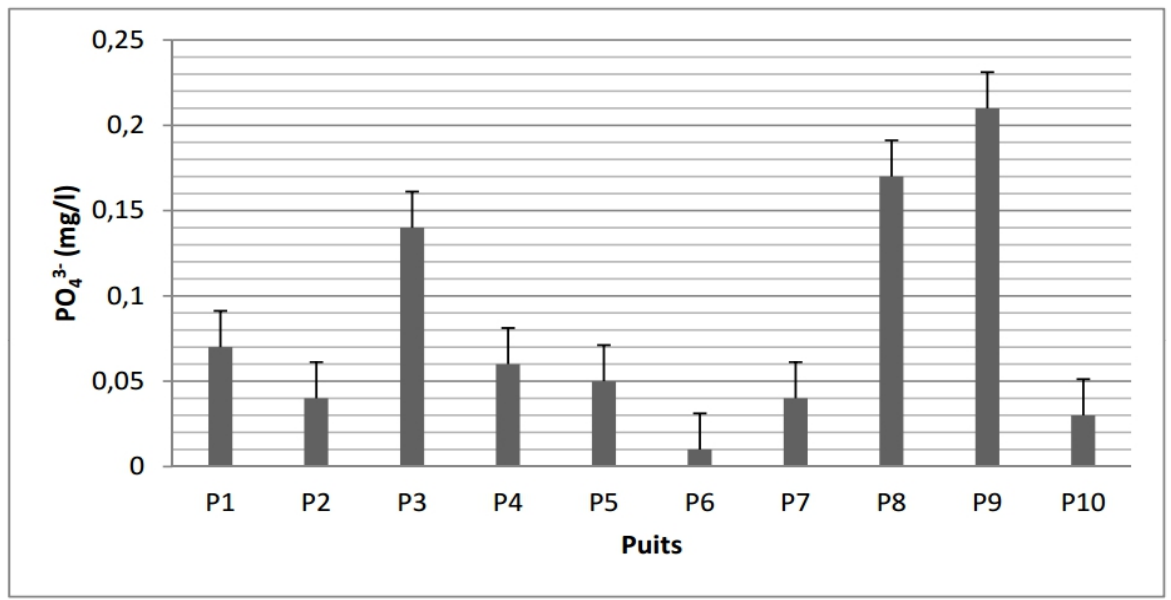

Figure 12: Variation des moyennes en Orthophosphates des eaux de puits 


\section{Caractérisation bactériologique}

Dans le domaine de l'hygiène, les analyses bactériologiques concernent souvent, non pas des micro-organismes pathogènes, mais des germes jouant un rôle d'indicateurs sans que leur présence constitue nécessairement un risque pour la santé publique. Ainsi deux types principaux d'indicateurs sont pris en considération: les indicateurs de contamination fécale permettant d'apprécier, avec plus ou moins de sûreté ou de précocité, le risque d'une contamination par des matières fécales pouvant véhiculer des micro-organismes pathogènes; les indicateurs d'efficacité de traitement permettant d'évaluer la qualité d'un traitement de désinfection de l'eau vis-àvis de micro-organismes pathogènes dont la présence peut être redoutée dans l'eau brute utilisée (NM 03.7.001. 2006).

Tableau 3: Résultats moyens de l'analyse bactérienne des eaux de puits

\begin{tabular}{|c|c|c|c|}
\hline Puits & Coliformes Fécaux & Coliformes Totaux & Streptocoques Fécaux \\
\hline P1 & 15 & 3 & 5 \\
\hline P2 & 0 & 0 & 0 \\
\hline P3 & 0 & 0 & 0 \\
\hline P4 & 0 & 0 & 0 \\
\hline P5 & 17 & 52 & 13 \\
\hline P6 & 200 & 69 & 20 \\
\hline P7 & 6 & 17 & 18 \\
\hline P8 & 1500 & 750 & 350 \\
\hline P9 & 150 & 240 & 1650 \\
\hline P10 & 2600 & 580 & 224 \\
\hline
\end{tabular}

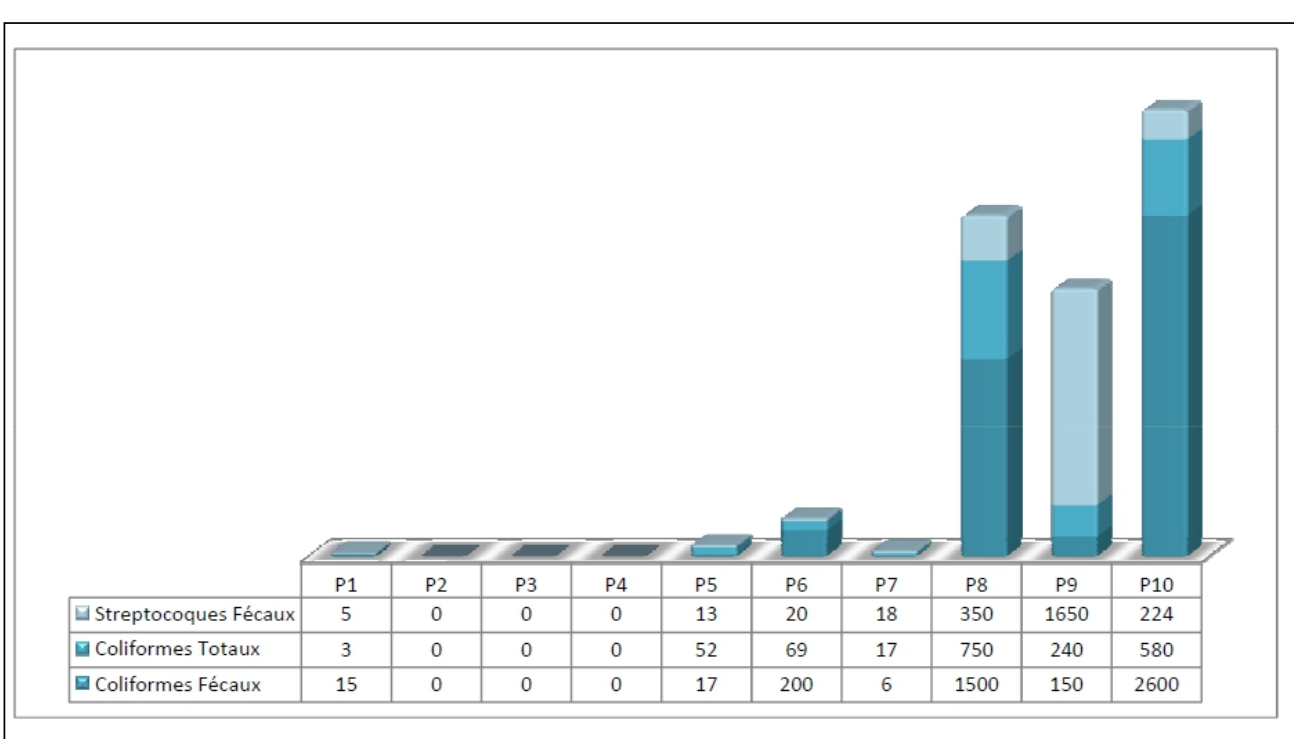

Figure 13 : Moyennes des analyses bactériennes des eaux des puits de Rhiss-Nekor 
- $\quad$ Pour qu'une eau soit prête à être consommé il est nécessaire que les bactéries soient absentes c'est le cas des puits P2, P3 et P4, par contre au niveau des autres puits on note la présence d'une quantité importante de bactéries par exemple P8 et P10 présente plus de 2000 bactérie des trois types réunis.

- $\quad$ Pour les coliformes totaux (CT), tous les points d'eau analysés sont contaminés (figure 13) à l'exception des puits P2, P3 et P4 dont la qualité bactériologique de ces eaux répond à la norme Marocaine (NM03.07.001, 2006) soit 0UFC/100ml. La valeur maximale des CT a été enregistrée dans les eaux du puits P8 (750UFC/100ml). Tous les puits étudiés sont utilisés pour desservir les populations avoisinantes en eau. Les résultats de cette étude ont montré que la quasi-totalité des puits sont contaminé par les CT, ce qui peut présenter un risque d'une contamination fécale. Cette contamination peut être causée par les rejets domestiques, par l'existence des puits à proximité des fosses septiques et d'une infiltration d'eau de surface dans les puits. Ces causes rejoignent celle détectées dans l'étude menée par (EI Haissoufi et al., 2011) sur la population des eaux de puits de certains quartiers de la ville de Fès au Maroc.

La recherche des coliformes fécaux (CF) dans les puits sélectionnés, a révélé que seulement les puits $\mathrm{P} 2$, P3 et $\mathrm{P} 4$ répondent à la norme marocaine (NM 03.7.001, 2006) (0UFC/100ml), les autres puits sont tous contaminés par ces germes. D’après les prospections dans le site des puits contrôlés, cette contamination serait due au fumier, aux fosses septiques, aux latrines et aux déchets de toutes natures existantes dans les terrains avoisinants les puits.

- $\quad$ La plus grande valeur moyenne des Streptocoques fécaux (SF) a été enregistrée (1650UFC/100ml) dans le puits P9) (Figure 13). La présence en nombre important de streptocoques fécaux dans les eaux de puits atteste la contamination des eaux par les matières fécales stockées dans les latrines.

\section{Conclusion}

A l'issue de cette étude qui a porté essentiellement sur la qualité physico-chimique et microbiologique des eaux de consommation, notamment les eaux de puits, il ressort que la quasi-totalité des paramètres analysés répond aux normes fixées par l’OMS. Néanmoins, L’analyse a révélé dans certains puits, qui sont au nombre de quatre, la présence de teneurs en nitrates anormales constituant ainsi, une pollution effective pouvant nuire à la santé du consommateur. Cependant, la qualité bactériologique montre une défaillance pour quelques puits étudiés, ceci constitue sans doute un risque pour la santé de la population consommatrice. Sur le plan bacteriologique, presque $70 \%$ des eaux de puits analysées sont 
souillées par les germes de contamination fécale et ne peuvent donc pas être consommées.

En dépit des valeurs des paramètres analysés qui restent dans les normes requises par l'OMS, il n'en demeure pas moins que ces différents puits d'eau considérés dans ce travail, constituent des sources potentielles de contamination au vue de leur statut et de leurs modes d'exploitation. Par conséquent, un programme d'aménagement, de traitement et d'assainissement de ces puits d'eau s'avère indispensable pour prévenir la population, de la zone d'étude, des maladies hydriques.

Dans l'absence de tout traitement, des études complémentaires seraient impératives pour chercher des paramètres de pollution plus dangereux tels que les pesticides et autres métaux lourds. Tous ces résultats montrent l'acuité du problème et la nécessité d’en limiter les effets. Il devient également urgent de rationaliser l'irrigation pour lutter efficacement contre cette pollution. Cette étude permettra une évaluation assez exhaustive des ressources en eau dans la plaine de Rhiss-Nekor et servira éventuellement d'outil d'aide à la décision.

\section{References:}

1. Abdoulaye Demba N'diaye, Khadijettou Mint Mohamed Salem, Mohamed Brahim El Kory, Mohamed Ould Sid' Ahmed OuldKankou, Michel Baudu. Contribution à l'étude de l'évolution Spatio-temporelle de la qualité physicochimique de l'Eau de la Rive droite du fleuve Sénégal, J. Mater. Environ. Sci. 5 (1) (2014) 320329.

2. Aka N., Bamba S.B., Soro N., Larhys. Journal 16 (2013) 31-52.

3. AMHARREF M., BERNOUSSI A. Vulnérabilité et risque de pollution des eaux souterraines. Actes des JSIRAUF, Hanoi, 6-9 novembre (2007).

4. Bermond R. et Perrdon C. Paramètres de la qualité des eaux. Ministère de l'environnement Paris, (1979) 259.

5. Belghiti. L, Chahloui A., Bengoumi D., El Moustaine R., Sci.Lib.Edit. Mersen. 5 (2013) 2-17.

6. Boualla N., Saad F.H., Sci. Lib. Edit. Mersen. 3 (2011) 1-10.

7. Brahimi. A., A. Chafi. Etude écotoxicologique de l’oued Za et de son affluent Oued Tizeghrane (Basse Moulouya, Maroc Oriental), J. Mater. Environ. Sci. 5 (5) (2014) 1671-1682.

8. Chapman D., Kimstach V. Selection of water quality variables.Water assessments : a guide to the use of biota, sediments and water in environment monitoring, Chapman edition, 2nd ed. E \& FN Spon, London, (1996) 59-126. 
9. Deliste C.E.et Schmidt J.W. The effects of sulphur on water and aquatic life in Canada.Dans: Sulphur and itsinorganic derivatives in the Canadian environnement, NRCC No. 15015, comité associé sur les critères

10. Degbey C., 2011. Facteurs associés à la problématique de la qualité de l'eau de boisson et la santé des populations dans la commune d'Abomey-calavi au Benin. Thèse de doctorat en Sciences de la santé publique. Ecole de santé publique. Université Libre de Bruxelles (ULB).

11. Derwich, L., Benziane, Z., Benaabidate, L. Diagnostic of physicochemical and bacteriological quality of fez wastewaters rejected in Sebou River: Morocco. Environ Earth Sci. Volume 63 (4), pp 839-846. 2011.

12. Dussart, B : Limnologie : Etude des eaux continentales. GauthierVillars, Ed., Paris, 1966. Scientifique concernant l'état de l'environnement, Conseil national de recherches du Canada, Ottawa (1977).

13. El Haissoufi H., Berrada S., Merzouki M., Aabouch M., Bennani L., Benlemlih M., Idir M., Zanibou A., Bennis Y., El Oualilami A., Microbiol. IND.San. ENVIRONN. 5 (1) (2011) 37-68

14. Gaujous D., La pollution des milieux aquatiques Aide-mémoire, $2^{\text {eme }}$ Ed Lavoisier. ISBN:2-7430-0021 (1995) 224p.

15. Guergazi S., Achour S., 2005. Larhyss Journal. 4, 119.

16. HCEFLCD: Etude sur la pisciculture au barrage Almassira, CR dar CHAFAAI, Cercle d'ELBROUGE, Province de Settat, 201p, 2006.

17. Laferriere, J.J. Minville, J. Lavoie et P. Payment, «L'industrie porcine et les risques à la santé humaine», Bull. Information Santé Environnement, Québec, 7 (2) (1996) 1-4.

18. Leynaud G. 1968. Les pollutions thermiques, influence de la température sur la vie aquatique. B.T.I. Ministère de l'agriculture, 224-881.

19. Ministère de l'Aménagement du Territoire, de l'eau et de l'Environnement (MATEE). Rapport sur l'Environnement au Maroc. Département de l'Environnement, Observatoire de l'Environnement, Octobre, Ed, 2, (2001) 296p.

20. Matthess G. Geol. Mijinbouw, 53, 194. -In Forstner U ET Wittmann GTW Metal pollution in the Aquatic Environment, (1994) 355-359.

21. NM 03.7.001. (2006). Norme marocaine relative à la qualité des eaux d'alimentation humaine. Bulletin Officiel N 5404 du 16 Mars 2006.

22. Nisbet E.G.et Verneau O. Composantes chimiques des eaux courantes, discussion et proposition de classes en tant que bases 
d’interprétation des analyses chimiques. Ann. Limn, 6(2) (1970) 161190.

23. Nouayti N., Khattach D., Hilali M., J. Mater. Environ. Sci. 6 (4) (2015) 1068-1081.

24. OFFICE NATIONAL DE L'EAU POTABLE (1997). Étude du schéma directeur d'assainissement liquide de la ville de Berrechid, Maroc. Rapport interne, $60 \mathrm{p}$.

25. OMS: directives de qualité pour les eaux de boisson; Volume 1Recommandation. Organisation mondiale de la santé $2^{\mathrm{e}}$ (1994)

26. Olduro, A.O. et Aderiye, B.1. 2007. Efficency of Moringa Oleiferasead extract on the microflora of surface and groundwater. J. Plant. Sci., 6: 453-438.

27. OCDE, 2001. Les perspectives de l'environnement (2001), Rapport de l'OCDE.

28. Pesson P. Pollution des eaux continentales, incidences sur les biocénoses aquatiques (1979) 97-101.

29. Polan Patrick et Monique Henry (1990) : qualité de l'eau souterraine dans la MRC de Coaticook, Régie régionale de la santé et des services sociaux de l'Estrie direction de la santé publique et de l'évaluation.

30. Rodier J. et al. L'analyse de l'eau, $9^{\mathrm{e}}$ édition. DUNOD (éditeur), Paris, France. (2009) 1579.

31. Rodier J. L'analyse de l'eau. Eaux naturelles, eaux résiduaires, eau de mer. $7^{\text {ème }}$ édition, Dunod, Paris, (1984) 1353.

32. Queneau P., and Hurbert J., Place of mineral water in the diet. Report of the National Academy of Medecine. Frensh Society of Medical Hydrology and Climatology, France, (2009) 175-220.

33. Servais P., Billen G., Garcia-Armisen T., George I.,Goncalvez A., Thibert S., 2009. La contamination microbienne dans le bassin de la Seine. Edition. Agence de l'Eau Seine Normandie. 50 pp. ISBN: 9782-918251-07-1 